\title{
Consultas por dolor torácico en el servicio de urgencia hospitalario de una comunidad rural. Llay-Llay 2005 - 2007
}

\author{
DANIEL MUÑOZ ${ }^{(1)}$, PABLO FUICA $^{(1)}$, NICOLÁS ALBERTZ $^{(1)}$ Y MARIA DE LA FUENTE $^{(2)}$
}

\section{RESUMEN}

El dolor torácico es una de las causas más frecuentes de consulta servicios de urgencia. Engloba causas banales y otras potencialmente mortales como las cardiovasculares.

Material y Método: Estudio descriptivo transversal. Se utilizaron fichas de atención y protocolos Auge de dolor torácico en consultantes por esta causa entre enero de 2005 y diciembre de 2007, en el servicio de urgencia del Hospital San Francisco de Llay-Llay.

Resultados: Sobre un total de 957 pacientes, el 48,69\% correspondió al sexo masculino y un 51,3\% al sexo femenino, con una media de edad de 50,16 años. Los diagnósticos más prevalentes fueron la costocondritis (37,5\%), trastorno ansioso (10,8\%), precordalgia inespecifica (8,5\%), crisis hipertensiva (6,6\%) y angina inestable (3,6\%). Cabe destacar un 2,4\% de casos de infarto agudo del miocardio, de los cuales el 73,9\% pertenecía al sexo masculino. Los principales factores de riesgo fueron la hipertensión arterial (39,7\%), obesidad (32,7\%) y tabaquismo (28,42\%). El consumo de sustancias se objetivó en un 33,99\%, principalmente alcohol y en el sexo masculino. La hospitalización alcanzó un 11,8\%, principalmente hombres de entre 61-80 años de edad, cuyo diagnóstico principal correspondió a la esfera cardiovascular.

Conclusión: En la gran mayoría de los casos el dolor torácico no representa gravedad, pero es fundamental descartar enfermedades serias, especialmente en pacientes con factores de riesgo. El diagnóstico y tratamiento oportuno deben basarse en un alto grado de sospecha del médico de urgencias, que cuenta con escasas herramientas, para lograr un diagnóstico específico y oportuno para mejorar el pronóstico de los pacientes que consultan por dolor torácico.

Palabras clave: Dolor torácico, factores de riesgo, urgencias, diagnóstico, infarto agudo del miocardio.

\section{ABSTRACT}

CONSULTATIONS WITH CHEST PAIN AS PRESENTING COMPLAINT AT THE EMERGENCY UNIT OF A RURAL COMMUNITY HOSPITAL IN LLAY-LLAY, BETWEEN 2005 AND 2007

Chest pain is one of the most common presenting complaint at the emergency units. It includes banal and potentially lethal causes such as cardiovascular ones.

\footnotetext{
(1) Facultad de Medicina. Universidad de Chile. Carlos Alvarado 6566. Las Condes. Santiago. Chile. danielito.m.s@gmail.com

(2) Escuela Salud Pública. Universidad de Chile.
} 
Materials and Methods: Cross-sectional descriptive study. Medical care records and AUGE protocols for chest pain in patients with chest pain as chief complaint attending the emergency unit at the Hospital San Francisco de LLay-Llay between January 2005 and December 2007 were used.

Results: Of a total of 957 patients, 48,69\% were males and 51,3\% were females, with a mean age of 50,16 years. The most prevalent diagnoses were costochondritis (37,5\%); anxiety disor$\operatorname{der}(10,8 \%)$; non-specific precordialgia (8,5\%), hypertensive crisis $(6,6 \%)$ and unstable angina $(3,6 \%)$. It is noteworthy that 2,4\% were acute myocardial infarction cases, of which $73,9 \%$ occurred in males. The main risk factors were high blood pressure $(39,7 \%)$, obesity $(32,7 \%)$ and smoking $(28,42 \%)$. Substance consumption was evidenced in 33,99\%, mainly involving alcohol and occurring in males. Hospital admission reached 11,8\%, involving mainly males aged between 61and 80, with diagnoses of cardiovascular conditions.

Conclusion: In the great majority of the cases chest pain did not represent a severe condition, however it is critical to rule out serious diseases, particularly in patients with risk factors. Timely diagnosis and treatment should be based on a high degree of suspicion from the emergency unit physician, who has seldom tools to attain a specific and timely diagnosis that will enable the improvement of the prognosis of patients attending with chest pain as chief complaint.

Keywords: Chest pain, risk factors, emergency, diagnosis, acute myocardial infarction.

\section{INTRODUCCIÓN}

El dolor torácico constituye una causa frecuente de consulta en servicios de urgencias tanto nacionales como internacionales. Representa entre $5 \%$ y $20 \%$ de las consultas de urgencia en hospitales generales ${ }^{1}$. Sin embargo, su importancia no radica sólo en este hecho, sino también en las nefastas consecuencias e impacto que conllevan algunos diagnósticos diferenciales, sobre todo los relacionados con la esfera cardiovascular ${ }^{2}$.

Resulta fundamental diferenciar aquellos pacientes que padecen enfermedades de manejo simple como la patología osteomuscular, el trastorno ansioso, la bronquitis aguda o la gastritis, de patologías graves como el síndrome coronario agudo, tromboembolismo pulmonar, disección aórtica, neumotórax a tensión, entre otros, que pueden comprometer la vida del paciente ${ }^{3}$.

En este sentido, se han elaborado diferentes protocolos que permitan al personal de salud diferenciar de manera rápida y efectiva la gravedad del cuadro para un correcto manejo oportuno, acorde a la patología en cuestión ${ }^{4}$.
Dichos protocolos evalúan elementos tales como sexo, edad, comorbilidad y factores de riesgo cardiovascular clásicos como hipertensión arterial, diabetes mellitus tipo 2 (DM2), obesidad, tabaquismo, dislipidemia, historia personal y familiar de eventos cardiovasculares, nunca olvidando el tipo de dolor, la historia clínica y el examen físico ${ }^{5}$. Asimismo, se han formado equipos multidisciplinarios para ingresar a estos pacientes en áreas específicas y especializadas, las llamadas Unidades de Dolor Torácico ${ }^{6}$.

Con el objeto de realizar una caracterización clínica y epidemiológica de los pacientes consultantes por dolor torácico en un servicio de urgencia de una localidad rural, se analizaron las atenciones de urgencia por esta causa en el Hospital San Francisco de Llay-Llay, ubicado en la Región de Valparaíso. Este hospital tiene a su cargo una población de 33.756 habitantes, incluyendo la vecina comuna de Catemu. Se analizó la presencia de factores de riesgo cardiovascular y consumo de sustancias, presencia de enfermedad cardiovascular previa, proporción de ingresos hospitalarios por esta causa y diagnóstico final de la atención. 


\section{MATERIAL Y MÉTODO}

Se realizó un estudio descriptivo transversal, en el cual se recabó la información contenida en fichas de atención de urgencia de pacientes que consultaron por dolor torácico entre el 1 de enero de 2005 y el 31 de diciembre del año 2007, en el servicio de urgencia (SU) del Hospital San Francisco de Llay-Llay. Dichas fichas contenían datos demográficos como sexo y edad, junto con una descripción breve de la historia clínica, examen físico, tratamiento y diagnóstico de egreso de cada paciente. Adicionalmente se registraron factores de riesgo cardiovascular, según el protocolo de dolor torácico Auge, el cual busca entregar elementos clínicos de sospecha para infarto agudo al miocardio (IAM).

Durante el periodo observado se consignaron 957 ingresos, cuya información fue registrada a través de una ficha electrónica elaborada con el programa Excel que contenía los siguientes datos: sexo y edad del paciente, factores de riesgo y antecedentes previos concernientes a patología cardiovascular como obesidad, tabaquismo, hipertensión arterial, DM2, dislipidemia, historia familiar, uso de nitritos, angina, IAM y bypass o angioplastía previos; consumo de sustancias (alcohol y drogas ilíci- tas), necesidad de hospitalización y diagnóstico de egreso del servicio de urgencia. Posteriormente, utilizando el programa Stata 8.0 se realizó el estudio de prevalencia de las diversas variables de la población consultante.

\section{RESULTADOS}

Sobre un total de 957 pacientes consultantes por dolor torácico, el 48,69\% $(n=466)$ correspondió al sexo masculino y un 51,3\% $(n=491)$ al sexo femenino. La media de edad de la muestra fue de 50,16 años (DE +/- 20,01) con un rango de 4 a 93 años.

El $33,75 \%$ de los pacientes se encontraba entre los 41-61 años, seguido por el 27,06\% entre 21 y 40 años y el 26,12\% entre 61-80 años de edad, como principales concentraciones por grupo etario.

En los diagnósticos de egreso desde el servicio de urgencia, se observa que la costocondritis aguda se alza como la causa más frecuente de consulta con un 37,5\% ( $\mathrm{n}=359)$, seguida del trastorno ansioso $(10,8 \% ; 103$ casos), precordalgia inespecífica $(8,5 \%$; 81 casos), crisis hipertensiva $(6,6 \% ; 63$ casos) y angina inestable $(3,6 \% ; 34$ casos) (Tabla 1$)$.

Tabla 1. Resumen de los diagnósticos finales más importantes de las consultas por dolor torácico en el servicio de urgencias.

\begin{tabular}{|l|c|c|}
\hline RESPIRATORIO & $\mathbf{N}^{\mathbf{0}}$ & $\mathbf{\%}$ \\
\hline Bronquitis aguda & 20 & $\mathbf{2 , 0 9}$ \\
\hline Neumonía & 13 & $\mathbf{1 , 3 6}$ \\
\hline Otros respiratorio $^{\mathrm{a}}$ & 16 & $\mathbf{1 , 6 7}$ \\
\hline PSIQUIATRICO & & $\mathbf{1 0 , 7 6}$ \\
\hline Trastorno ansioso & 103 & $\mathbf{0 , 4 2}$ \\
\hline Crisis conversiva & 4 & \\
\hline $\begin{array}{l}\text { CARDIOVASCULAR } \\
\text { Trastornos del ritmo cardiaco }\end{array}$ & & $\mathbf{7 , 1}$ \\
\hline Crisis hipertensiva & 68 & $\mathbf{6 , 5 8}$ \\
\hline
\end{tabular}

(Tabla continúa en página siguiente) 


\begin{tabular}{|l|c|c|}
\hline Angina Inestable & 34 & $\mathbf{3 , 5 5}$ \\
\hline Angina Estable & 28 & $\mathbf{2 , 9 2}$ \\
\hline IAM & 23 & $\mathbf{2 , 4}$ \\
\hline Otros cardiovascular ${ }^{\mathrm{b}}$ & 23 & $\mathbf{2 , 4}$ \\
\hline & & \\
\hline OSTEOMUSCULAR & 359 & $\mathbf{3 7 , 5 1}$ \\
\hline Costocondritis & 10 & $\mathbf{1 , 0 4}$ \\
\hline Contusión torácica & 17 & $\mathbf{1 , 7 7}$ \\
\hline Otros osteomuscular ${ }^{\mathrm{c}}$ & & $\mathbf{2 , 9 3}$ \\
\hline & & $\mathbf{1 , 3 6}$ \\
\hline DIGESTIVO & 28 & $\mathbf{0 , 9 4}$ \\
\hline Gastritis & 13 & $\mathbf{1 , 3 6}$ \\
\hline Esofagitis & 9 & \\
\hline Espasmo esofágico & 13 & $\mathbf{9 . 2 9}$ \\
\hline Otros digestivo & \\
\hline OTROS & & $\mathbf{1 , 0 4}$ \\
\hline Precordalgia inespecífica/no isquémico & 89 & $\mathbf{0 , 7 3}$ \\
\hline Etilismo agudo & 10 & $\mathbf{0 , 7 3}$ \\
\hline Síndrome vertiginoso & 7 & $\mathbf{1 0 0} \%$ \\
\hline Otros & 7 & $\mathbf{9 5 7}$ \\
\hline TOTAL & & \\
\hline
\end{tabular}

${ }^{a}$ Asma, faringitis aguda, neumotórax, etc.

${ }^{\mathrm{b}}$ Angina de Prinzmetal, edema pulmonar agudo, etc.

${ }^{\mathrm{c}} \mathrm{Hombro}$ doloroso, mastalgia, etc.

${ }^{\mathrm{d} C o ́ l i c o ~ b i l i a r, ~ g a s t r o e n t e r i t i s ~ a g u d a, ~ e t c . ~}$

Según sistemas comprometidos, encontramos las causas osteomusculares con un 40,3\% $(\mathrm{n}=386)$; seguidos de la esfera cardiovascular con el $24,97 \%(n=239)$ y problemas asociados a salud mental con el 11,18\% $(n=107)$. Es importante señalar que en el 9,09\% $(\mathrm{n}=87)$, sólo se pudo descartar el origen isquémico del dolor, sin precisar la causa específica (Tabla 2).

Tabla 2. Distribución de los diagnósticos, en número y porcentaje, agrupados por sistema.

\begin{tabular}{|l|l|l|}
\hline & $\mathrm{N}^{\circ}$ & $\%$ \\
\hline Osteomuscular & 386 & 40,33 \\
Cardiovascular & 239 & 24,97 \\
Trastornos Ansiosos & 107 & 11,18 \\
Precordalgia inespecífica & 87 & 9,09 \\
Digestivo & 63 & 6,58 \\
Respiratorio & 49 & 5,12 \\
Otros* & 26 & 2,71 \\
\hline Total & $\mathbf{9 5 7}$ & $\mathbf{1 0 0}$ \\
\hline
\end{tabular}

*Incluye pacientes que referían dolor torácico no clasificables, con etilismo agudo o DM2 descompensada. 
Detectamos consumo de sustancias en el $33,99 \%(\mathrm{n}=193)$ de la muestra. De estos, el consumo de alcohol representó el 95,13\%, principalmente de sexo masculino (62,5\%). Del mismo modo, encontramos consumo de Cocaína (5 casos), Marihuana (3 casos) y Pasta Base (1 caso).

Los principales factores de riesgo cardiovascular en la población en general fueron la hipertensión arterial $(39,7 \%)$, la obesidad $(32,7 \%)$ y el tabaquismo $(28,42 \%)$. Se constató el antecedente de dislipidemia y DM2 en un $11,9 \%$ y $9,5 \%$ respectivamente. En este mismo ámbito un $10,97 \%$ presentaba antecedentes familiares de enfermedad cardiovascular, 5,85\% angina previa, 3,65\% IAM anterior, 3,03\% uso de nitritos y $1,66 \%$ presentaba angioplastía o bypass coronario (Figura 1).

Figura 1. Incidencia de factores de riesgo y su distribución por sexo en la muestra de pacientes.

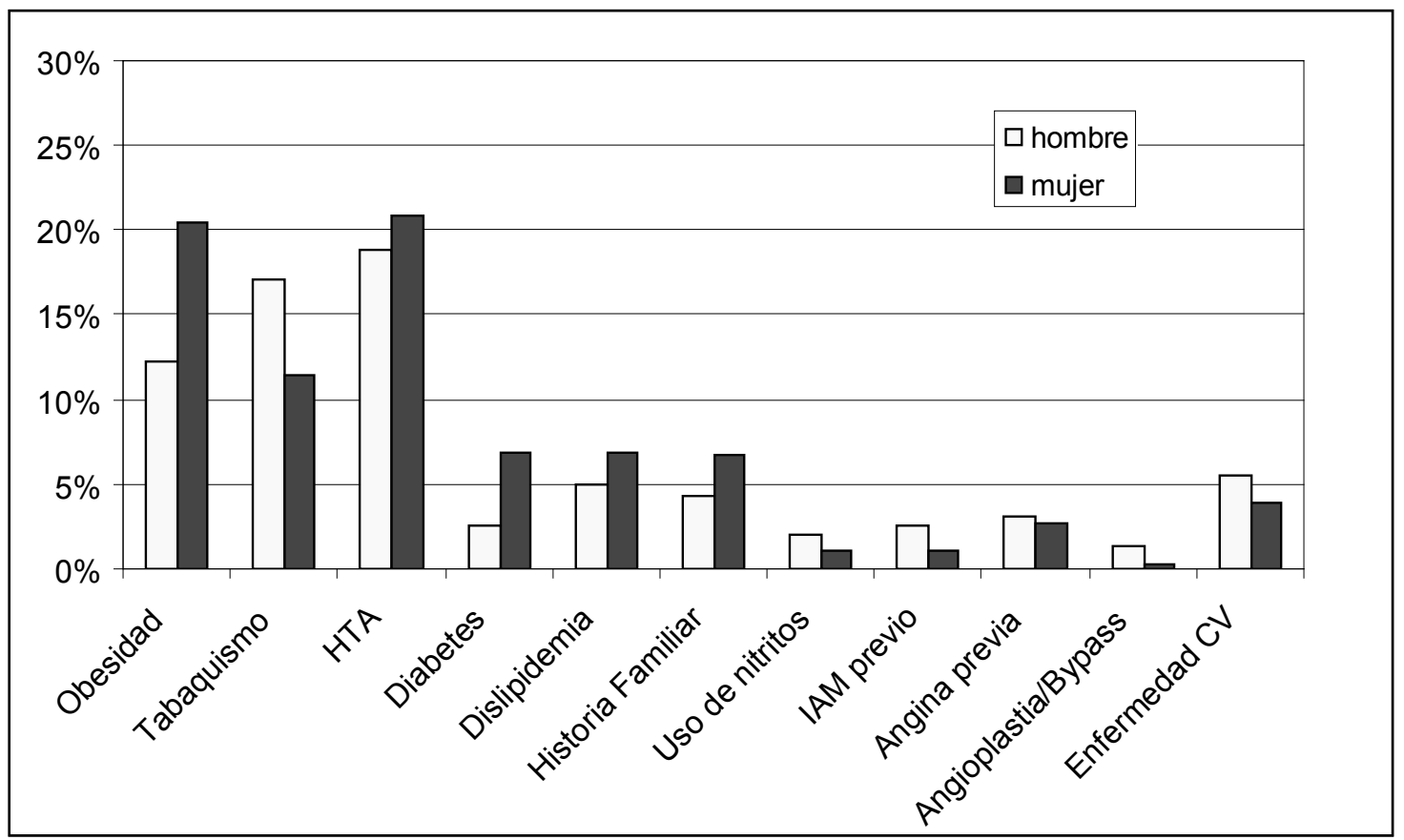

Se precisó el ingreso hospitalario en el $11,8 \%(\mathrm{n}=113)$ de la muestra estudiada, en la mayoría de los casos pacientes de sexo masculino (62,83\%), ubicados principalmente en el rango de 61-80 años de edad. El diagnóstico principal en este grupo correspondió a la esfera cardiovascular, principalmente aquellas entidades incluidas en dentro de la cardiopatía coronaria, destacando la angina inestable y el IAM con un $28,31 \%$ y $19,46 \%$ respectivamente del total de hospitalizados. Le siguen las arritmias cardíacas con un $15,04 \%$ y las crisis hipertensiva con un $6,19 \%$.
Según sexo, observamos que en ambos sexos el diagnóstico de egreso más importante fue la costocondritis $(31,1 \%$ en hombres y $43,6 \%$ en mujeres), seguida de la precordalgia inespecífica en hombres $(11,2 \%)$ y del trastorno ansioso en mujeres (13\%). En cuanto a factores de riesgo, la hipertensión arterial se alza en ambos casos como el más frecuente $(38,6 \%$ hombres; $40,7 \%$ mujeres). En el grupo de sexo masculino el segundo lugar lo ocupa el tabaquismo (35\%), seguido de la obesidad $(27,1 \%)$ y dislipidemia $(10,3 \%)$. Para el grupo de mujeres, tanto la obesidad $(39,9 \%)$ como la DM2 $(13,4 \%)$ ocupan 
un lugar más importante como factor de riesgo (Figura 1). El índice de hospitalización (15,2\% hombres; $8,6 \%$ mujeres) y el consumo de sustancias (principalmente alcohol con $22,7 \%$ en hombres y $13,4 \%$ en mujeres) fue mayor en el sexo masculino.

Según grupo etario, se encontraron los resultados que se resumen en la Tabla 3. Para el grupo de entre 0 y 20 años de edad, los diag- nósticos más frecuentes fueron la costocondritis aguda con el 51,51\%, seguido de los trastornos ansiosos con un 18,18\%. El índice de hospitalización fue de un 3,03\% y el consumo de sustancias alcanzó un 10,6\%. Entre los factores de riesgo destacan el tabaquismo con un $28,78 \%$, seguido de la obesidad con un $18,18 \%$ y la historia familiar de enfermedad cardiovascular con un $10,6 \%$.

Tabla 3. Factores de riesgo y diagnósticos más importantes según grupo etario.

\begin{tabular}{|c|c|c|c|c|}
\hline Grupo etario (años) & Factores de riesgo & $\%$ & Diagnósticos & $\%$ \\
\hline \multirow[t]{3}{*}{0 - 20} & Tabaquismo & 28,78 & Costocondritis & 51,51 \\
\hline & Obesidad & 18,18 & Trastorno ansioso & 18,18 \\
\hline & Historia familiar & 10,6 & & \\
\hline \multirow[t]{4}{*}{$21-40$} & Tabaquismo & 42,85 & Costocondritis & 49 \\
\hline & Obesidad & 23,9 & Trastorno ansioso & 13,1 \\
\hline & HTA & 11,2 & Precordalgia inespecífica & 10,8 \\
\hline & Historia familiar & 9,3 & & \\
\hline \multirow[t]{4}{*}{$41-60$} & HTA & 42,1 & Costocondritis & 30,95 \\
\hline & Obesidad & 38,39 & Trastorno ansioso & 9,59 \\
\hline & Tabaquismo & 30,03 & Precordalgia inespecífica & 7,43 \\
\hline & Otros & 33,06 & Otros & 12,07 \\
\hline \multirow[t]{4}{*}{$61-80$} & HTA & 68,8 & Costocondritis & 26 \\
\hline & Obesidad & 46,1 & Síndrome coronario agudo & 16,4 \\
\hline & Angina/IAM & 22,4 & Crisis hipertensiva & 10 \\
\hline & Otros & 37,6 & Otros & 14,4 \\
\hline \multirow[t]{4}{*}{$>80$} & HTA & 72,88 & Síndrome coronario agudo & 23,7 \\
\hline & $\begin{array}{l}\text { Enfermedad } \\
\text { Cardiovascular }\end{array}$ & 25,4 & Arritmias & 22 \\
\hline & Obesidad & 18,64 & Insuficiencia Cardiaca & 10,1 \\
\hline & & & Otros & 16.87 \\
\hline
\end{tabular}

En el grupo entre 21 y 40 años tenemos la costocondritis aguda con un $49 \%$, los trastornos ansiosos con el 13,1\% y precordalgia inespecífica (descartada la causa isquémica) con un 10,8\%. En este grupo aparece el síndrome co- ronario agudo como entidad diagnóstica (incluyendo angina estable, inestable e IAM) con un $2,31 \%$ de incidencia. En este grupo se hospitalizó un 5,01\% y se observó consumo de sustancias en el 16,9\%. Los principales factores de riesgo 
fueron el tabaquismo con el 42,85\%, la obesidad con el 23,9\%, la hipertensión arterial con el $11,2 \%$ y la historia familiar de enfermedad cardiovascular con una incidencia del 9,3\%.

En pacientes entre 41 y 60 años de edad encontramos costocondritis aguda en un 30,95\%, los trastornos ansiosos con un $9,59 \%$, la precordalgia inespecífica con un 7,43\%, los síndromes coronarios agudos con un $6,5 \%$ y la crisis hipertensiva con 5,57\%. Un 7,73\% de los consultantes de este grupo requirió hospitalización, destacando el fallecimiento de 1 paciente en el servicio de urgencia producto de un paro cardiorrespiratorio secundario a IAM. El consumo de sustancias alcanzó un 20,7\%. Los factores de riesgo más importantes para este grupo fueron la hipertensión arterial con un $42,1 \%$, la obesidad con un $38,39 \%$, el tabaquismo con el $30,03 \%$, dislipidemia con el $16,71 \%$, la DM2 con el 9,59\% y antecedentes familiares de enfermedad cardiovascular con un $16,4 \%$. Destaca la aparición de factores de riesgo como la angina y el IAM previos con un $5,26 \%$ y $1,5 \%$ respectivamente.

Para la población entre 61 y 80 años de edad, encontramos costocondritis aguda con un $26 \%$, seguido por cardiopatía isquémica (angi- na estable e inestable y IAM) con un $16,4 \%$, la crisis hipertensiva (10\%), la precordalgia inespecífica $(8 \%)$, los trastornos ansiosos $(6,4 \%)$ y la fibrilación auricular (6,4\%). El 21,2\% de los casos tuvo necesidad de hospitalización y el consumo de sustancias estuvo presente en un $18,4 \%$, dado en su totalidad por consumo del alcohol. Los principales factores de riesgo para este subgrupo fueron la hipertensión arterial con un $68,8 \%$, obesidad con $46,1 \%$, IAM y angina previa juntos con un $22,4 \%$, DM2 con un $21,2 \%$ y dislipidemia con un $16,4 \%$.

En el grupo de pacientes mayores de 80 años hallamos el síndrome coronario agudo en un $23,7 \%$, alteraciones del ritmo cardíaco en conjunto con un $22 \%$, la insuficiencia cardiaca descompensada y costocondritis aguda (ambas con un $10,1 \%$ ) y el trastorno ansioso con un $6,77 \%$. Para este grupo etario el índice de hospitalización alcanzó un 33,89\% y se detectó consumo de sustancias en un 16,9\% (únicamente alcohol). Los principales factores de riesgo fueron la hipertensión arterial con un 72,88\%, el antecedente de enfermedad cardiovascular y la obesidad con un $25,4 \%$ y $18,64 \%$ respectivamente. La Figura 2 esquematiza el porcentaje de hospitalización para cada grupo etario del estudio.

Figura 2. Índice de hospitalización según grupo etario.

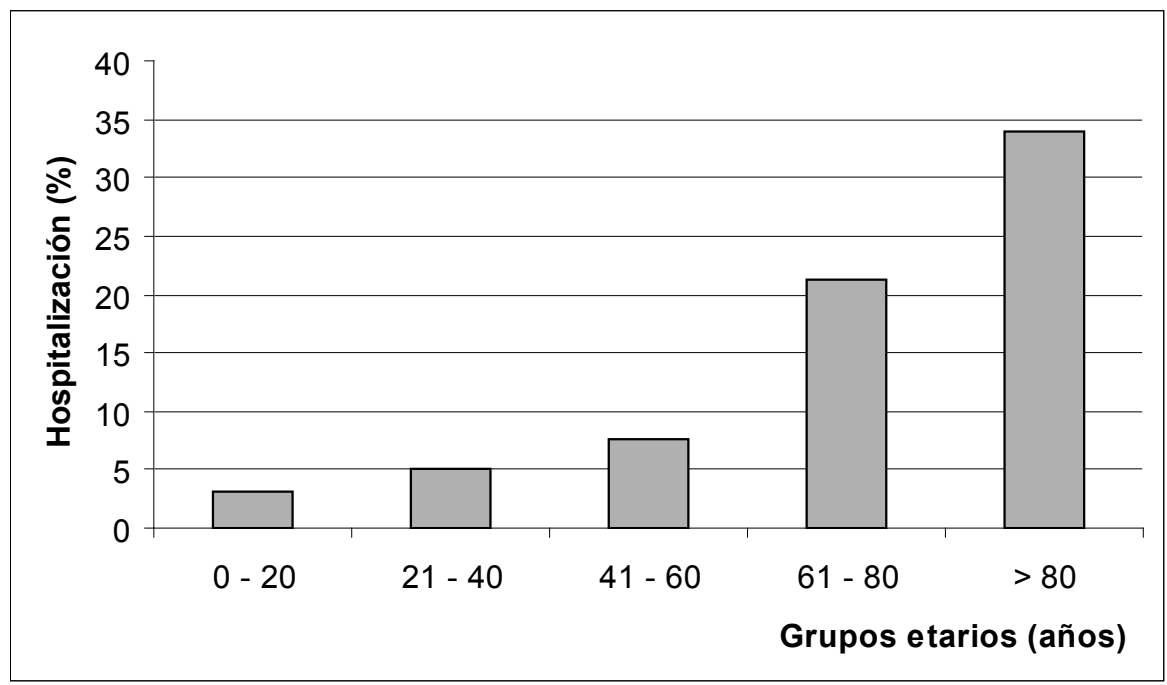


Los casos de IAM correspondieron al 2,4\% de las consultas (23 casos). La media de edad de fue de 60,26 años (DE +/- 15,03) con un rango de 27-91 años. El 73,9\% (n=17) de los afectados pertenecía al sexo masculino. Los factores de riesgo dentro de este subgrupo de pacientes fueron el tabaco con un 47,8\%, hipertensión arterial con el $43,5 \%$ y el antecedente de angina con $34,8 \%$. El 21,7\% (5 casos) presentaba el antecedente de un episodio de IAM.

\section{DISCUSIÓN}

El dolor torácico es un motivo de consulta que afecta a todos los grupos etarios y de manera similar a ambos sexos. Hemos observado que un mismo síntoma puede manifestar una amplia variedad de patologías que afectan prácticamente a todos los sistemas del organismo, incluyendo la esfera psiquiátrica, cardiorrespiratoria, digestiva y osteomuscular, lo que está ampliamente documentado en la literatura científica $^{4,6,7}$.

El principal diagnóstico de egreso del servicio de urgencia fue la costocondritis aguda o Síndrome de Tietze, entendiendo por ello a aquel dolor de las uniones esternocostales, de carácter benigno y resolución espontánea. Le siguen en frecuencia los trastornos ansiosos y la precordalgia inespecífica (aquel dolor torácico no clasificable, habiendo descartado la etiología isquémica a través de electrocardiograma). La costocondritis aguda prevaleció en ambos sexos y en todas las edades, exceptuando los mayores de 80 años. Este hecho puede tener especial relación con el tipo de trabajo que realiza la población, principalmente labores agrícolas o relacionadas con la construcción. Creemos que posiblemente exista cierto grado de abuso diagnóstico en cuanto a costocondritis aguda, dada su predominancia en casi todas las edades, lo que puede tener relación con la presión asistencial o la falta de herramientas diagnósticas. En este sentido, cabe señalar que de acuerdo al protocolo ministerial de evaluación de dolor torácico, la única herramienta discriminatoria está enfocada al uso de electrocardiograma, con apoyo de telemedicina en su interpretación ${ }^{5}$. Además, creemos que la inclusión de etilismo agudo y síndrome vertiginoso como diagnóstico final, se deba posiblemente a la presencia de una precordalgia inespecífica en concomitancia con alguna patología que distrae al clínico de urgencias.

En los demás factores se produjeron variaciones según el perfil demográfico de los pacientes, predominando el trastorno ansioso en la población más joven (menor de 40 años) y la cardiopatía coronaria, en la población mayor de 60 años. Estos hechos son concordantes con reportes similares sobre etiología del dolor torácico ${ }^{8-10}$.

Al agrupar estos diagnósticos por sistemas, observamos nuevamente el predominio de la patología osteomuscular (dado principalmente por la costocondritis), sin embargo, la patología cardiovascular en su conjunto, incluyendo la cardiopatía coronaria, hipertensiva, insuficiencia cardiaca y trastornos del ritmo, constituyó la segunda causa de consulta a nivel global.

Según sexo, observamos que si bien ambos grupos comparten muchos factores de riesgo, siendo la hipertensión arterial el principal en ambos grupos, los demás factores analizados presentan una incidencia distinta, predominando el tabaquismo en el caso de los hombres y la obesidad en las mujeres. En éstas últimas se encontró además mayor prevalencia de DM2. En los diagnósticos según sexo, vemos el predominio entre los hombres de la cardiopatía coronaria, mientras que en mujeres se observa mayor prevalencia de los trastornos del ritmo cardíaco. En cuanto al consumo de sustancias, este está dado predominantemente por el alcohol en ambos sexos, sin embargo, los hombres superan casi por el doble la prevalencia de consumo en el sexo femenino. El porcentaje de hospitalización también fue mayor en el sexo masculino. 
Como se dijo anteriormente, es importante destacar que en un alto porcentaje no se pudo determinar un diagnóstico específico luego de descartar el origen isquémico del dolor, hecho también destacado en la literatura revisada ${ }^{11-14}$. Otra limitación importante que hemos detectado tiene que ver con la imposibilidad de realizar seguimiento de los pacientes, para la confirmación definitiva de su diagnóstico y evolución, dada la baja complejidad del hospital de esta localidad rural.

El principal factor de riesgo en la población general lo constituyó la hipertensión arterial, seguida de la obesidad y el tabaquismo. La primera, aumentó a medida que aumentaba la edad de los pacientes, desde un $11,2 \%$ en pacientes entre 21 a 40 años, hasta un $72,8 \%$ en pacientes mayores de 80 años. Esto se observó también en el caso de la obesidad, el antecedente de cardiopatía coronaria (incluyendo IAM) y la DM2. En el caso del tabaco, existe mayor prevalencia entre los más jóvenes.

La necesidad de hospitalización estuvo dada principalmente por patología cardiovascular, concentrada principalmente en la población masculina entre los 61 y 80 años, lo que coincide con estudios similares en poblaciones también parecidas ${ }^{15,16}$.

El alcohol fue la sustancia más consumida, fluctuando en los distintos grupos etarios entre el 16 y $20 \%$. Se registró una prevalencia marginal de drogas ilícitas (marihuana, cocaína y pasta base).

Al analizar los casos de IAM, al 2,4\% de las consultas (23 casos), predominando en el sexo masculino $73,9 \%$; siendo los principales factores de riesgo el tabaquismo y la hipertensión arterial. Un número no despreciable de pacientes presentaba antecedentes de angina e IAM. Si bien promedio de edad de ocurrencia fue de 60 años, se registró un amplio rango de edad (27 a 91 años), lo que indica la importancia de sospechar este diagnóstico a cualquier edad ${ }^{17,18}$. Posiblemente sean necesarios estudios que separen a los adultos de las edades pediátricas para obtener una caracterización más fidedigna de poblaciones cuyas patologías se comportan de manera disímil.

En un estudio de Castro et $\mathrm{al}^{6}$, si bien está enfocado a clasificar a los pacientes según probabilidad de presentar un síndrome coronario agudo, se logra conocer la magnitud del dolor torácico en un hospital universitario y se consigue establecer la importancia de una adecuada aplicación de los protocolos de evaluación. Al igual que en nuestro estudio, se identifican loas factores de riesgo cardiovascular más importantes, siendo la HTA la primera en frecuencia, a pesar de ser poblaciones con estilos de vida distintos.

Finalmente, la importancia de estudios como éste tiene especial valor si consideramos que se trata de un motivo de consulta muy frecuente y que eventualmente involucra patologías graves en cuyo manejo se gastan importantes cantidades de dinero, lo que pone al médico de atención primaria en una posición privilegiada para diagnosticar a tiempo, lo que mejora el pronóstico y las alternativas que se pueden ofrecer a estos pacientes.

Podemos concluir, que en la gran mayoría de los casos, el dolor torácico no es sinónimo de una enfermedad grave, sin embargo, deben descartarse diagnósticos tales como el IAM sin importar la edad, sexo o ausencia de factores de riesgo. Para esto, debemos realizar una anamnesis, examen físico y exámenes complementarios que incluyan como mínimo un electrocardiograma, para detectar aquella patología que pueda representar una amenaza para la vida del paciente. Estas medidas permiten un manejo adecuado y oportuno, que mejoran el pronóstico de los pacientes que ingresan al servicio de urgencia.

Recibido: 11 julio 2008

Aceptado: 1 oct. 2008 


\section{REFERENCIAS}

1. MEISEL JL. Diagnostic approach to the patient with chest pain. UpToDate ${ }^{\circledR}$ 13.1, 2005.

2. CAYLEY W. Diagnosing the Cause of Chest Pain. Am Fam Physician 2005;72:2012-21.

3. ANDERSON E, GLAUSER J. Non-cardiac causes of chest pain in the emergency department. Emergency Medicine Reports 2003; 24: 221-8.

4. FOX K. Investigation and management of chest pain. Heart 2005; 91:105-110.

5. Ministerio de Salud. Guía Clínica Infarto Agudo del Miocardio y Manejo del Dolor Torácico en Unidades de Emergencia. 1st Ed. Santiago: Minsal, 2005.

6. Castro P, Corbalán R, Isa R, Gabrielli L, Perez O, Chamorro $\mathrm{G}$ et al. Unidad de dolor torácico: primera experiencia en Chile. Rev Méd Chile 2007; 135: 839-845

7. Sanchis J, Bodí V, Núñez J, Bosch X, LomaOsorio P, Mainar L et al. Limitations of clinical history for evaluation of patients with acute chest pain, non-diagnostic electrocardiogram, and normal troponin. Am J Cardiol. 2008 Mar 1;101(5):613-7.

8. Lee TH, Goldman L. Evaluation of the patient with acute chest pain. N Engl J Med 2000; 342: 1187-95.

9. Fang J, Bjorkman D. A critical approach to noncardiac chest pain: pathophysiology, diagnosis, and treatment. Am J Gastroenterol. 2001;96: 958-968.

10. Huffman J, Pollack M. Predicting Panic Disorder Among Patients With Chest Pain: An Analysis of the Literature. Psychosomatics 2003; 44:222-236.
11. Fass R. Chest pain of esophageal origin. Curr Opin Gastroenterol. 2002;18:464-470.

12. France J, Keen C, Bowyer S. Communicating risk to emergency department patients with chest pain. Emerg Med J. 2008 May;25(5):276-8.

13. Gorenberg M, Marmor A, Rotstein H. Detection of chest pain of non-cardiac origin at the emergency room by a new non-invasive device avoiding unnecessary admission to hospital. Emerg Med J. 2005 Jul;22(7):486-9.

14. Ortiz-Olvera NX, González-Martínez M, RuizFlores LG, Blancas-Valencia JM, Morán-Villota $\mathrm{S}$, Dehesa-Violante M. Causes of non-cardiac chest pain: multidisciplinary perspective. Rev Gastroenterol Mex. 2007 Apr-Jun;72(2):92-9.

15. Bassan R, Gibler WB. Unidades de dolor torácico: estado actual del manejo de pacientes con dolor torácico en los servicios de urgencias. Rev Esp Cardiol 2001; 54: 1103-9.Fesmire FM, Campbell M,

16. Decker W, Howell JM, Kline JA. Clinical policy: critical issue in the evaluation and management of adult patients presenting with suspected acute myocardial infarction orunstable angina. Ann Emerg Med 2000; 35: 251-4.

17. Hagberg SM, Woitalla F, Crawford P; American College of Cardiology; American Heart Association. 2002 ACC/AHA guideline versus clinician judgment as diagnostic tests for chest pain. J Am Board Fam Med. 2008 MarApr;21(2):101-7.

18. Ruiz-Ros V, Sanchis-Forés J, Bodí-Peris V, Núñez-Villota J, Gómez-Monsoliu C, BoschCampos MJ et al. Predictive value of chest pain score for the diagnosis of acute coronary syndromes. Med Clin (Barc). 2006 Jan $14 ; 126(1): 1-4$.

Usted puede comentar éste y otros artículos publicados en la Revista Chilena de Salud Pública, enviando un correo electrónico a revistasp@med.uchile.cl 